\title{
Application of Imitation Control and Foot Sensors to Bipedal Robot Walking
}

\author{
Hee-Jung Byun ${ }^{1}$ and Su Goog Shon ${ }^{1 *}$ \\ ${ }^{1}$ Department of IT Engineering, University of Suwon, IT Building, San 2-2 Wau-ri, \\ Bongdam-eup, Hwaseong-si, South Korea \\ ${ }^{1}$ heejungbyun@suwon.ac.kr, ${ }^{1}{ }^{*}$ sshon@suwon.ac.kr
}

\begin{abstract}
In this paper a method that allows for the control of a humanoid robot with foot sensors through an imitation control approach is presented. Our approach does not use any prior analytical or mathematical robot modeling (e.g. forward or inverse kinematics). The key concept is to trace the CoP (center of pressure) as fast as possible and to control the ankle of the robot's support foot prior to impact. The experimental results demonstrate that a humanoid robot can successfully imitate the motions of the operator and can walk stably by utilizing both the CoP and controlling the robot's ankle.
\end{abstract}

Keywords: Bipedal robot, control communication, CoP, foot sensor, imitation control

\section{Introduction}

It has been expected that the number of application areas of robots could be increased with the remote control of robots. In general, robots do simple and dangerous jobs in the place of people. For instance, they are engaged in simple assembly processes in manufacturing plants, exploration of the deep sea, and dismantling explosives.

Flexible mobility and intelligence are very much required for specific robotics applications. Since N. Tesla demonstrated the first radio-controlled boat in 1898 and R. Goertz demonstrated a bilateral manipulator in 1954 [7], remotely operated robots have been widely designed. Teleoperation for a robot is control from a secure distance by a human operator, and at the same time, the robot should avoid being harmed by incorrect user commands (self-protection) and avoid causing damage (avoid collisions). Finally the teleoperation should try to provide some feedback to the operator and try to automatically identify incidents, sending back information to the user.

In order to enhance the mobility of robots, wheeled robots have been considered. Wheeled robots, even though they possess superior mobility, have difficulty moving over debris. In addition, wheeled robots cannot themselves decide how to get through those kind of obstacles because of their low intelligence. So, teleoperation technology has been adopted to compensate for intelligence. Research concerning the teleoperation of robots have usually been done in wheeled robots such as Pioneer 3AT, Erratic EraMobi, and Surveyor SRV-1 Blackfin [14].

We use humanoid robots because their anthropomorphic design makes them suitable for interacting in human environments and intuitive for humans to operate. Humanoid robots are well suited to using human tools, closing valves, opening doors, climbing staircases, and so forth. Humanoid robots have advantages over wheeled robots in that their human-like form allows for them to take advantage of urban environments designed by people for people.

* Corresponding Author 
Humanoids have better mobility than conventional wheeled robots, but they tend to tip over easily.

Even though many studies all around the world have been done to develop humanoid robots, their intelligence remains insufficient to perform many tasks. It will not soon be possible for humanoids to be sufficiently intelligent to operate independently. One possible solution is to control humanoid robots by teleoperation.

Many studies for the development of humanoids robots are currently underway. Studies for robots closer to humans in appearance, face expression and gait, and for technology development are underway. Honda ASIMO is the most representative example [16].

Since Honda began to develop humanoid robots in 1986, there have been attempts to teleoperate bipedal robots. Remote controllers (RC) for flight vehicles were first used for bipedal robots, but it turned out that they are low in DOF and possessed limited motion control. Various types of robots able to meet consumer needs have been designed with the ability to be controlled via wired/wireless communication. In order to teleoperate a humanoid, several technologies such as console, joy stick, special interface, motion capture suit, etc., have been utilized.

With a teleoperation console, an operator inputs the robot's path and actions such as power on, stand by, simple tasks, and power off. The robot is then able to move on a planned path autonomously and to perform simple operations via wireless teleoperation, as system developed in 1998 [2-3]. A joystick master device was also developed 2002 for the teleoperation of whole body motions of humanoid robots [4]. Using a joy stick with the assumption that the operator understands the environment, the operator can then move a humanoid robot with only a single three-axis joystick with a button via teleoperation, which provides directional commands to be interpreted. Here, the robot computes trajectories that satisfy kinematic and dynamic constraints to safely execute the desired motion [6]. Robots in LRM Lab. have been teleoperated by humans with remote controllers and a communication network since 2009 [1]. Keio University has shown that bipedal robots can be controlled by recognizing touch inputs of gestures similar to those used in touch-screen smartphones [24]. A. Senior and S. Tosunnoglu have presented how to control bipedal robots by transmitting commands from a computer with a serial communication connection to a bipedal robot [25]. Additionally, Bluetooth is can be used to control a robot remotely. In terms of real-time operating system for robot, European automotive operating standard (OSEK/VDX) has also been introduced in order to increase the reliability and stability of the robot [28].

\section{Related Works}

Early approaches to the teleoperation of humanoid robots captured user intention through simple devices such as joysticks, buttons, and keyboards. Advances in robotic technology have brought about sophisticated humanoid robots. The necessity to teleoperate humanoids by a human operator has remained even as robotics technology has advanced. One approach has been to employ a "marionette" system, consisting of a small scale puppet version of the humanoid robot [9] for manipulation by the operator. A drawback of this approach is the need for purpose built hardware, which in some cases can be large, cumbersome, and difficult to transport.

As humanoid robot control requires a large degree of freedom for dexterous tasks, the main challenges concern how to best satisfy the operator's desired behavior for the robot given the (dimensional) differences between the input capture device and the robot, while also maintaining the robot's stability. 
A human-controlled, humanoid robot that employs imitation control would be intelligent enough to perform difficult jobs because it is controlled by a human's intelligence. Because this type of imitation-controlled robot with mobility and intelligence is attractive, there is an increasing amount of research in this area.

Imitation control means that the human operator's joint movements are traced and transmitted to the robot so it can move like the human operator. Moreover, the development of imitation devices for humanoid robots has presented challenging research issues. To trace body movements, gyroscope and acceleration sensors are attached to specific areas of the human body.

Motion capture has had a significant impact on robotics, being used for not only teleoperation but also for improving humanoid robots. Early approaches used inertial measurement units [10], photo detectors [11,23], and shape tape [12]. In recent years, many new motion capture products have come onto the market, ranging from the cheap but somewhat limited (e.g. Microsoft Kinect, Nintendo Wii) to the (relatively) expensive but highly accurate Xsens MVN full-body motion capture suit [8].

In one instance, a master interface worn by human operator that served to map the lower limb joint angles of the human operator to those of the humanoid robot was designed and obtained rough terrain adaptability by means of teleoperation in 2004 [5]. T. Liu et al. especially have presented that a robot's gait can be estimated after applying a fuzzy inference engine to measurement data of angular speed and acceleration $[17,18]$. Ude et al. has used a magnetic marking systems for the measurement of joint angles [19]. A small inertial sensor system has also been used to measure lower body segment orientations [20]. R. Chalodhorn et al. have said that direct use of motion capture data is more likely to be flexible and intuitive than programming control, though it is kinetically unstable [21]. As another instance, both a real-time 3D human motion capture system using force sensors and a hand tracking device can be used for full-body motion reconstruction [22].

Even though there are different types of sensor systems to trace the motion or joint angles of the human operator, they are not direct methods, and as a result require some calculations from indirect measurements such as optical tracking, magnetic marks, acceleration sensors, or a real time method.

Even though there have been some studies concerning imitation control, most of have not yet been applied to actual robot walking. Only virtual robots in cyberspace have yet been able to imitate an operator's motions well. Imitation control could be used for the more-difficult components of a task, leaving the rest to autonomy, or as a fallback option if autonomy fails.

The main problem of the imitation control is that the walking stability of a robot is not guaranteed because the joint angles of the robot are generated by the human operator. Unseen trajectory data can cause instability in robot's walking gait. This paper proposes a new method to resolve the walking instability caused by imitation control. The key issue in this paper is to control the walking stability for a bipedal robot in real time. If sensing and changing the walking status of a robot is possible in real time, accomplishing a stable gait may be possible. In order to verify this assumption, some experiments have been done to show the possibility of real-time control of the posture of a robot. In the following sections, how fast a robot can fall down is studied by using an inverted pendulum model and the falling time of an actual robot is measured. Finally, experiments show that an imitation-controlled robot can be controlled with the help of fast control . 


\section{Imitation Control System and Robot Kinematics}

Imitation control is a well-known method for bipedal robot walking, which uses the operator's motion data to control a robot's movement. This paper studies challenging issues to overcome the postural instability caused by dynamics of robots.

The proposed imitation control system is shown in Figure 1. The control system consists of a bipedal robot, a controller, and a wearable encoding system. The bipedal robot can be controlled so as to imitate the motions carried out by the operator.

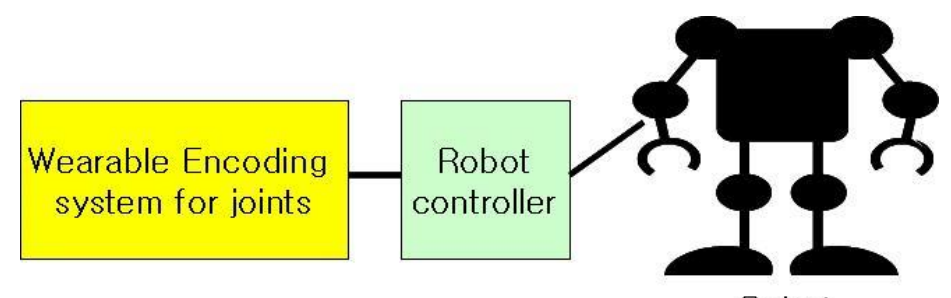

Robot

Figure 1. Imitation Control System for a Bipedal Robot

The wearable encoding system is used to obtain trajectory data as the human operator moves. The operator's gait and walking patterns obtained are transmitted to the robot controller via a communication link. As for the robot controller, these data for walking trajectory are transmitted to the robot's actuator controllers.

The imitation control system can be simplified as in Figure 2. The robot controller serves the position angles for the operator's joints $\theta_{\mathrm{o} 1}, \ldots, \theta_{\mathrm{om}}$, which represent the operator's arm, leg, and body posture, respectively, to correspond to their robot counterparts $\theta_{\mathrm{r} 1}, \ldots, \theta_{\mathrm{rm}}$.

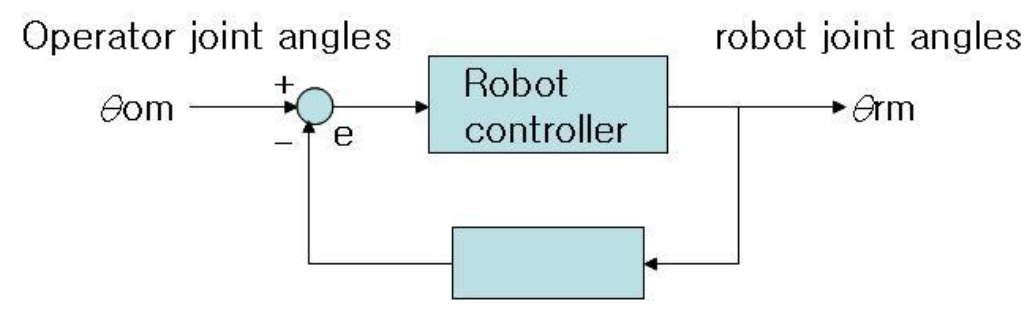

Figure 2. Simplified Model for Imitation Control System

Theoretically, imitation control can be done easily by copying the operator's joint angles to the robot's corresponding joints as in the equation (1). It is assumed that there is negligible data transfer time between the operator and the bipedal robot.

$$
\theta_{o 1} \approx \theta_{r 1}, \theta_{o 2} \approx \theta_{r 2}, \ldots, \theta_{o m} \approx \theta_{r m}
$$

From the view of robot kinematics, both forward and inverse kinematic solutions are generally required to control a robot via teleoperation.

Traditional approaches to humanoid teleoperation use explicit forward kinematic modeling of both the robot slave and master device to provide mapping between human motion capture data and robot actuator commands. Forward kinematics modeling involves calculating the position and orientation of the end-effector in three dimensional space given the joint angles (e.g. calculating the position of the hand in XYZ space based upon the values of the shoulder, 
elbow, and wrist joints). This is easily solved using the geometry of the robot which is specified in the DH parameters.

Conversely, inverse kinematics refers to the use of the kinematics equations of a robot to determine the joint angles given the desired position and orientation of the end-effector. Often, multiple solutions exists for the robot's inverse kinematic calculation if multiple configurations of the robot's joints can result in the same end-effector position. One approach to solving inverse kinematics is through trigonometry, while another is to use Jacobian matrix-based iterative approaches [13]. Both approaches are laborious and changes in robotic hardware require new analysis and kinematic calculations. Another approach to achieve the teleoperation of a humanoid robot such that it is able to execute simple tasks is to use a closed-form inverse kinematics and a feedback controller with the HUBO $2+$ platform, which was done as a DARPA project in 2013. The solution to this inverse problem involves solving for the joint angles given a desired position and orientation while accounting for singularities, joint limits, and feasible workspace issues [15].

Modeling and solving kinematics problems can be laborious, hardware specific, and sometimes computationally expensive. As for traditional imitation control, inverse kinematics problems have to been solved. In this paper, there is no need to solve inverse kinematic problems to find out the joint angles of each of arm and leg. We explore how to use an imitation control with foot sensors and allow an operator to command gaits without worrying about kinematics, leaving those tasks to the humanoid robot. For autonomy, foot sensors are planned to provide walking stability. In the following section, more details are discussed. The key benefits of our approach is that it provides flexibility and intelligence.

\section{Calculation and Estimation of Falling Time}

First, the time required for a robot to fall to the ground was investigated. The reason for this was to acquire some information pertaining to how fast the robot should be controlled.

Let us consider the robot as an inverted plane pendulum in a homogeneous gravitational field with acceleration g. As shown in Figure 3, the length of the pendulum is L, its mass is $\mathrm{m}$, its angular position is $\theta$, and its moment of inertia is given by equation (2).

$$
\mathrm{J}=\mathrm{mL}^{2} \text {. }
$$

The dynamical equation of motion of the pendulum is reduced to the angular momentum equation (3)

$$
J \frac{d \omega}{d t}=m g L \sin \theta
$$

where $\omega$ is the angular velocity defined by equation (4).

$$
\omega \equiv \frac{d \theta}{d t}
$$

Solving the differential equation for time, the formula for the calculation of the falling time of the pendulum is provided by equation (5) [27]

$$
T_{\text {fall }}\left(\theta_{0}, \omega_{0}\right)=\sqrt{\frac{L}{2 g}} \int_{\theta_{0}}^{\pi / 2} \frac{d \theta}{\sqrt{\frac{\omega_{0}^{2} L}{2 g}+\cos \theta_{0}-\cos \theta}}
$$


where $\theta_{0}$ is the pendulum's initial position and initial angular velocity $\omega_{0}=\omega(0)$. The result is expressed in terms of first order elliptic integrals.

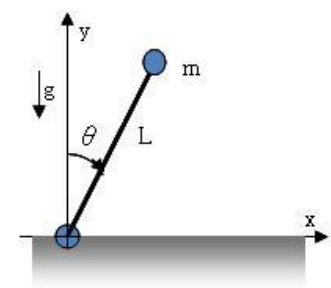

\section{Figure 3. Pendulum Model for Humanoid Robots}

For example, let $\mathrm{L}=0.25[\mathrm{~m}], \mathrm{g}=9.8\left[\mathrm{~m} / \mathrm{s}^{2}\right]$, and $\omega_{0}=0$. Then one have $\sqrt{\frac{L}{2 g}} \approx 0.1129$ so the falling time $T_{\mathrm{f} \text { all }}$ for an initial inclination of $\theta_{0}=1^{0}$ becomes 0.827 [sec]. The falling times are calculated and listed in Table 1 with different initial inclinations by the MATLAB program. The falling times are calculated as less than $1[\mathrm{sec}]$.

Table 1. Calculated Falling Times for the Inverted Pendulum

\begin{tabular}{|c|c|}
\hline Initial inclination $\theta_{0}$ & $\begin{array}{c}\text { Falling time } \mathrm{T}_{\text {fall }} \\
\text { [sec] }\end{array}$ \\
\hline $0^{0}$ & $\infty$ \\
\hline $1^{0}$ & 0.827 \\
\hline $2^{0}$ & 0.7219 \\
\hline $4^{0}$ & 0.6177 \\
\hline $8^{0}$ & 0.5166 \\
\hline $15^{0}$ & 0.4306 \\
\hline $30^{0}$ & 0.3483 \\
\hline
\end{tabular}

From the above calculations, the robot falls down very quickly, the speed of which depends on the initial inclination of the pendulum.

Some experiments to investigate the falling time of an actual bipedal robot have been performed. A three-axis acceleration sensor of Control Electronics Ltd. was used for measurement. The acceleration sensor was attached to the bipedal robot and the falling time and patterns were measured for several initial inclination angle $\theta_{0}$. Figure 4 shows the shape of the robot's foot, which affects the falling time.

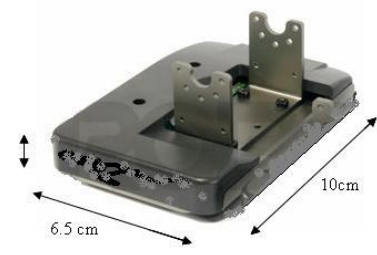

Figure 4. The Shape of the Foot

Figure 5 shows an acceleration signal for $1^{0}$ of initial inclination, where the $\mathrm{x}$ and $\mathrm{y}$ axes represent time and acceleration speed, respectively. The acceleration values remain extraordinary after touching the ground because the sensor is rotated $90^{\circ}$. 


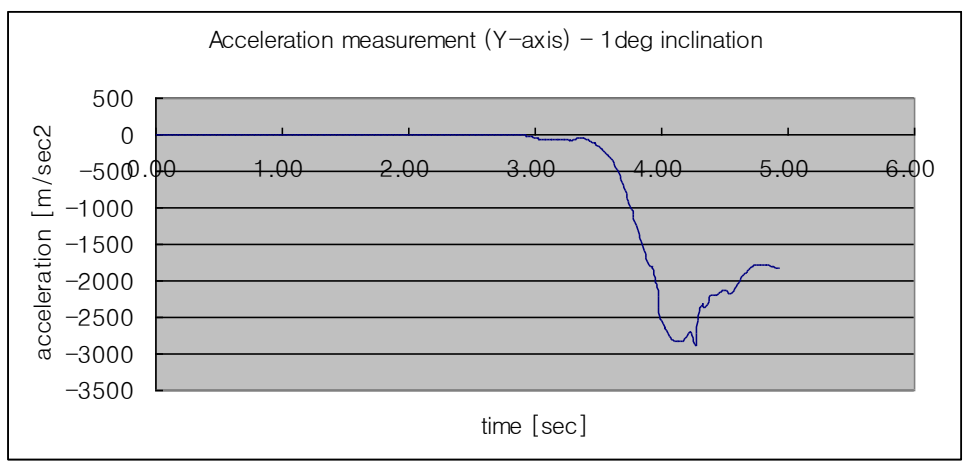

Figure 5. Acceleration for Initial Inclination Angle of 10

Table 2 lists the measured falling times for the actual bipedal robot. Experiments show that the actual bipedal robot falls down a little bit slowly rather than the inverted pendulum model predicts. A bipedal robot with a wide foot as shown in Figure 4 quickly begins to fall down after about $450 \mathrm{~ms}$ and finally touches the ground after about $1.15 \mathrm{sec}$ for an initial inclination of $1^{0}$.

\section{Table 2. Recorded Falling Times for the Bipedal Robot}

\begin{tabular}{|c|c|}
\hline Initial inclination $\theta_{0}$ & $\begin{array}{c}\text { Falling time } \mathrm{T}_{\text {fall }} \\
{[\mathrm{sec}]}\end{array}$ \\
\hline $0^{0}$ & $\infty$ \\
\hline $1^{0}$ & 1.15 \\
\hline $2^{0}$ & 1.0 \\
\hline $4^{0}$ & 0.9 \\
\hline $8^{0}$ & 0.85 \\
\hline $15^{0}$ & 0.73 \\
\hline $30^{0}$ & 0.62 \\
\hline
\end{tabular}

From the above calculations, the robot falls down very quickly, the speed of which depends on the initial inclination of the pendulum. Also, from some experiments involving the use of an acceleration sensor, the falling times are verified to range from few tens of milliseconds to around half second required to fall down the ground. However, it is known that a human operator cannot control a robot within the allowable time limits. It takes some time for the operator's brain to perceive what is occurring and to move the operator's hands, arms, or legs as a response. Saul Sternberg presented human perception and reaction time as around $400 \mathrm{~ms}$ [26]. Also, he said that this delay time varied from person to person.

The traditional robot moves according to a pre-designed trajectory with stability by using ZMP or static walking algorithms. However, in imitation control, the robot moves according to an unplanned trajectory. The most challenging issue is to overcome and resolve unstable movement with a fast identification and control method for gait stability.

\section{Imitation Control Algorithm}

Traditionally, autonomous robots' walking is kinematically pre-designed to be stable. A series of movements with precalculated postures are executed. However, for teleoperated imitation control, the joints' angles generated by the operator's motions are not predictive. Unexpected or random joint values can be created depending on the robot's environment. If 
such random joint data are directly entered into the robot, it cannot maintain its kinematic balance. The moment when the bipedal robot lifts one foot to start walking, it begins to fall down due to unstable posture. It is also hard to control the robot's posture balance remotely in real time due to temporal delay.

Let's suppose that a robot walks on a flat surface. The robot's ankle joints are assigned to control its balance because the ankle is the pivot point of an inverted pendulum. In addition, the other joints are assigned for determining the robot's direction and posture. So, the robot's joints are separated into two groups: balance and posture. The robot's joints that relate to posture are triggered by the operator's motion, while the ankle joints that relate to balance are controlled by the controller.

By controlling the robot as an inverted pendulum, a static walking process is applied in this paper. Dynamic walking process as expressed in equation (3) is a further research topic. The robot is controlled to be positioned within a static equilibrium area. Even though unforeseen joint angles are employed as the robot walks, it needs to be controlled in the equilibrium area as in equation (6), which keeps the robot stable.

$$
\text { Center of Mass }=\sum_{i=1}^{N} \frac{m_{i} \mathbf{I}_{i}}{M} \leq \text { Equilibrium area }
$$

,where $I_{i}$ is the position of joint, $m_{i}$ the weight of joint, and $M$ is the total weight of all joints.

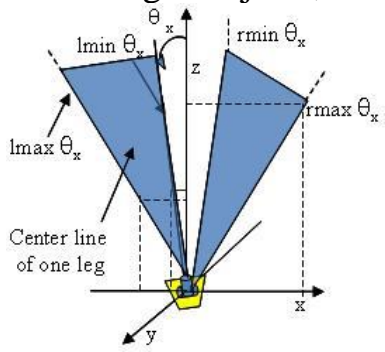

Figure 6. The Equilibrium Area for both the Left and Right Directions

The ankle joints of the foot suppress back and forth rocking movement, and cause left and right rocking to be damped down. The ankle joints function as pivot points and have 2 degrees of freedom (DOF): the left-right and back-forth directions. The equilibrium area for the leftright direction, as shown in Figure 6, is experimentally measured as follows. The robot's ankle joints for left-right movement are rotated against the direction of the other leg so as not to fall down when one leg is lifted up. The rotation angle varies dependant on the robot's posture such as how much the robot's leg leans or where the body, arms, or legs are positioned. The lmin $\theta \mathrm{x}$ and $\operatorname{lmax} \theta \mathrm{x}$ are measured to be $10^{\circ}$ and $15^{\circ}$, respectively, from experiments. In the same way, rmin $\theta \mathrm{x}$ and rmax $\theta \mathrm{x}$ are $10^{\circ}$ and $15^{\circ}$, respectively.

In the same way, the equilibrium area for the front-back direction can be measured. The foot's ankle is moved up to $10^{\circ}$ forwards or $15^{\circ}$ backwards, and the ankle moved in the opposite direction of the other ankle so as not to fall down when the body is moved forwards or backwards.

Based on the above experimental equilibrium area, walking procedures are designed as shown in Figure 7. After the operator is aware of the terrain around the robot, the operator plans where it will go. Joint motion signals are produced in the encoder as the operator moves, and transferred into the controller. The controller does not transmit data directly to the joint actuators, but transmits to individual joints after applying the walking control algorithm.

The point of the control algorithm is that it makes the robot's ankle joints remain within the equilibrium area. In this way, it can achieve posture balance without falling down. After that, 
the robot's posture or gait is established. Walking speed must be slow so the dynamic inertial force exerted by the robot can be ignored.

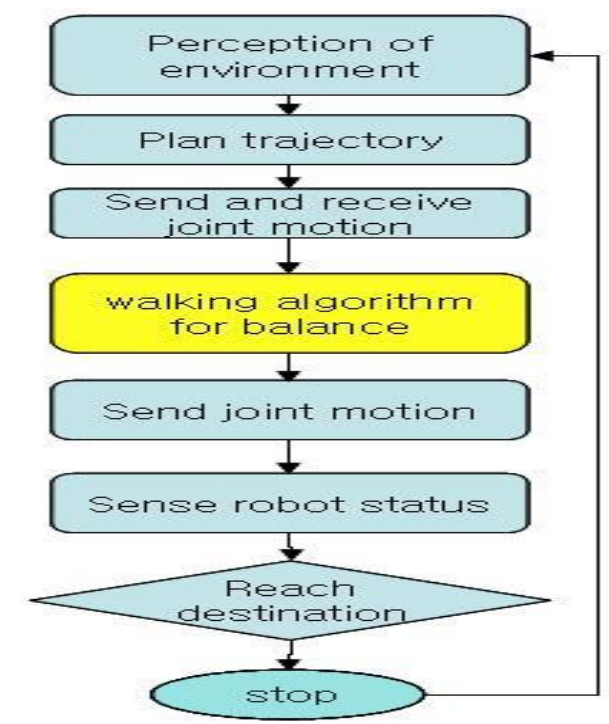

Figure 7. Walking Procedures for Balance

\section{Wearable Encoding and Foot Sensor System}

\subsection{Wearable Encoding System}

Wearable sensing systems are attracting extensive interest as a lower-cost human-robot control system without constraints from stationary motion analysis devices. One such wearable encoding system is described in Figure 8. This encoding system consists of a resistive type of rotary encoder, a strap, and a data processing module. The strap is designed to attach the encoding system to the human joints. As the operator moves his joint, the wearable encoding system attached generates a joint angular signal. The signal is sent to the data processing unit in which it is read by the microcontroller and transferred to the communication port. The signal from the resistive type of rotary encoder is converted into a digital signal for microcontroller processing.

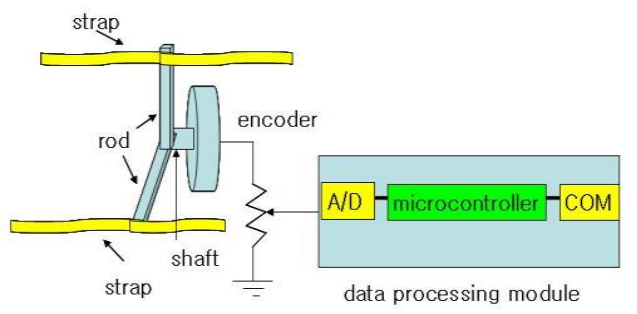

Figure 8. Wearable Encoding System

This wearable encoding system is implemented by integrating an 8-bit Atmel AVR microcontroller (ATmega8A, power consumption of $3.6 \mathrm{~mA}$ at $3 \mathrm{~V}$ ) and an incremental rotary encoder. The ATmega8A chip has $8 \mathrm{~K}$ Bytes of flash program memory and $1 \mathrm{~K}$ Byte of internal SRAM. The encoder can work with low energy consumption and is 
suitable for ambulatory measurements. Figure 9 shows the data processing unit of the rotary encoder.

The wearable encoding system can be attached to the hip, thigh, and knee. The joint angle values are obtained for the all the sensor via the communication port.

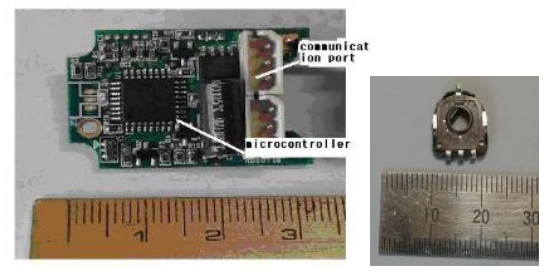

Figure 9. Encoder Sensor Module

An experiment was done on a flat floor in a usual office environment in order to evaluate the accuracy and performance of the wearable encoding system. Figure 10 shows the comparison of angular motions between for the robot's joint and the operator's joint. The experiment indicates how the robot can imitate as the operator moves his joint position randomly. It was observed that the robot (dotted line) follows the operator (solid line) well and there is a small temporal delay between the operator and the actuator.

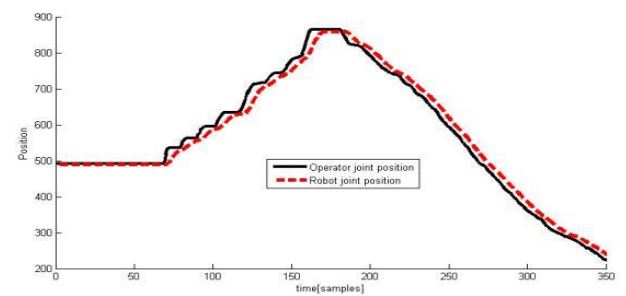

\section{Figure 10. Comparison for joint angle motion}

\subsection{Foot Sensor System}

One thing to keep in mind is that the operator needs to know the robot's situation. Even though the robot's joints may move according to the operator's joints, a sensing system like a foot sensor is required for the measurement of walking status.

The purpose of a foot pressure sensing system is to measures the center of pressure (CoP). The foot pressure sensing system consists of the resistive type of force sensor and a data processing module. As the posture of the robot changes, the pressure values for pressure sensors are also changed. The calculated $\mathrm{CoP}$ is also changed.

The foot pressure sensing system, as shown in Figure 11, is implemented by integrating a 32bit Cortex microcontroller and an incremental pressure sensor. A thin tactile force sensor, FlexiForce ${ }^{\circledR}$, by Tekscan Inc. is used for force or pressure measurement. The Cortex (myCortex-LM308) chip has $16 \mathrm{~K}$ Bytes of flash program memory and $4 \mathrm{~K}$ Bytes of internal SRAM. The pressure signals are sent to the data processing unit in which it is read by the microcontroller (Cortex) and transferred to the communication port for further processing. Four pressure sensors (P1, P2, P3, and P4) are used for each foot. 

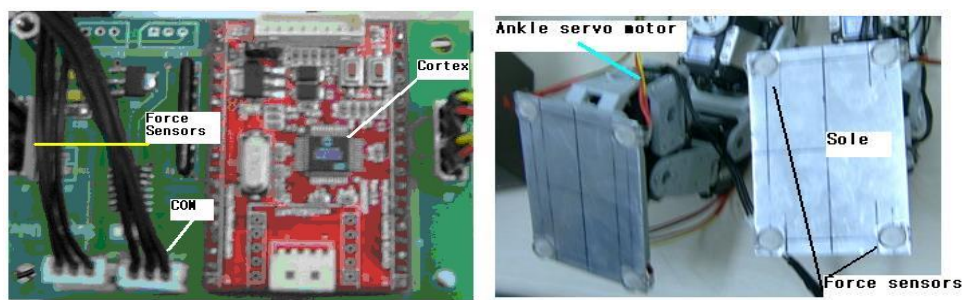

Figure 11. Implemented Foot Pressure Sensing System

\section{Experiments and Results}

We have tested the walking motions with an actual robot on a flat surface. Our experimental results are shown in Figure 12. The bipedal robot used in this paper has a total of 17 servomotor joints in its body. It has 17 DOFs (Degree of Freedom) including vertical vibration (pitch), rotation (yaw), and horizontal vibration (roll) DOF. The 17 DOFs of the bipedal robot are 10 for its legs, 6 for its arms, and 1 for its waist. ROBOTIS's Dynamixel AX-12A servomotors are used for the joints. The experimental results demonstrate that the humanoid robot can successfully imitate a series of human walking motions. The operator lets the robot move or stop as he wishes. However, the movement is very slow due to being unstable. A static walking algorithm based on $\mathrm{CoP}$ was utilized to perform a few scenarios to learn how well the robot imitates the operator.

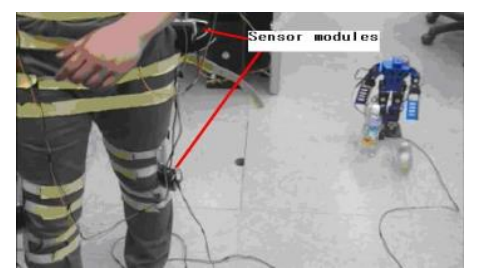

Figure 12. Imitation Control with a Wearable Sensor

The joint angles are transferred to a software application running on a personal computer. A custom built $\mathrm{C}$ software application running on the computer performs data preprocessing. To find a mapping between human movement and robot movement, motion data for both the robot and the human need to be collected and synchronized.

In order to show the possibility of controlling a robot in real time, the $\mathrm{CoP}$ and pressure values for the foot sensors are measured and controlling of the ankle joints is done within 0.5 [sec]. Before the robot falls down, it is possible to establish a stable gait through control of the ankle joint servomotors. Figure 13 describes pressure values on a foot sensor that can be utilized to control the target robot's gait through the ankle joint of the supporting leg.

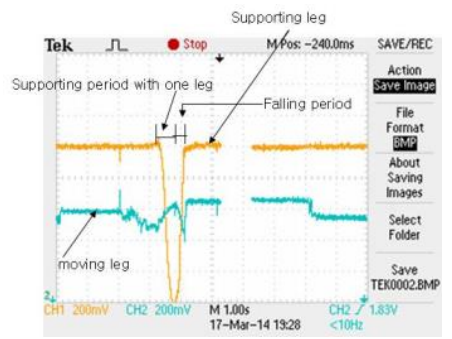

Figure 13. Changes of Pressure Signals 
Figure 14 a) and b) describes how the $\mathrm{CoP}$ is traced in case of stable walking and falling down, respectively.

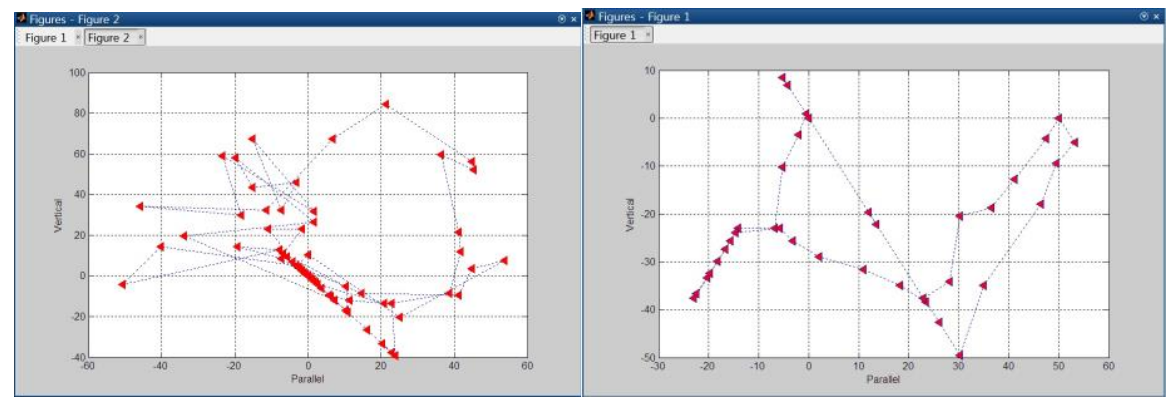

Figure 14. Trace of CoP in Case of a) Stable Walking b) Falling Down

\section{Conclusions}

Robot walking by using imitation control approach is very useful and convenient because generation of gait and trajectory data for the robot can be produced easily. However, the joint angles generated by the operator's motions are not predictive and cause instability for the postural gait of the robot. For instance, when a foot stands with one leg, the angle of the ankle servomotor is controlled until the robot establishes a stable gait.

With the proposed imitation control based on $\mathrm{CoP}$ and ankle joint control, the robot walking is done stably without the use of any a priori analytical or mathematical modeling. Characteristics of the imitation control should be improved by further research. To improve robot walking speed, research for the application of dynamic walking is underway.

Ideally such a system would allow individual users to quickly and easily tailor their chosen movements and gestures for accurate and intuitive control of any robotic system. Our work can make progress towards applications in hazardous rescue or construction.

\section{Acknowledgements}

This study was conducted by [GRRC (Gyeonggi Regional Research Center) Suwon 2014-B4, Developing technology and systems for coping with situation] as one of projects of the GRRC, Gyeonggi Province.

This article is a revised and expanded version of a paper entitled Control Communication and Application of Foot Sensor on Imitation presented at the 2014 International Conference on Future Information \& Communication Engineering held on June 26-28, 2014, in Kowloon, Hong Kong.

\section{References}

[1] G. Pessin and F. S. Osorio, "Genetic Algorithm Applied to Robotic Squad Coordination", IEEE Press /IEEE Computer Society, vol. 1, (2009), pp. 1-6.

[2] H. Takanobu, E. Guglielmelli, H. Tabayashi, S. Narita, A. Takanishi and P. Dario, "Remote Interaction between Human and Humanoid Robot", IARP First International Workshop on Humanoid and Human Friendly Robotics, (1998).

[3] K. Hirai, M. Hirose, Y. Haikawa and T. Takenaka, "The development of Honda humanoid robot", Robotics and Automation, Proceedings, vol. 2, (1998), pp. 1321 - 1326.

[4] N. E. Sian, K. Yokoi, S. Kajita, F. Kanehiro and K. Tanie, "Whole Body Teleoperation of a Humanoid Robot Development of a Simple Master Device using Joystics", Proceedings of the IEEE/RSJ, (2002).

[5] W. Y. Sim, C. M. Chew and G. S.Hong, "The use of teleoperation for humanoid walking: a first look", Robotics, Automation and Mechatronics, IEEE Conference on, vol. 1, (2004), pp. 1-6.

[6] M. Stilman, K. Nishiwaki and S. Kagami, "Humanoid Teleoperation for Whole Body Manipulation", Proceeding of the IEEE Int. Conf. on Robotics and Automation, (2008). 
[7] R. Goertz and R. Thompson, "Electronically controlled manipulator", Nucleonics, vol. 12, no. 11, (1954), pp. 46-47.

[8] C. Stanton, A. Bogdanvoych and E. Ratanasena, "Teleoperation of a humanoid robot using full-body motion capture, example movements, and machine learning", Proceedings of Australasian Conference on Robotics and Automation, (2012).

[9] T. Takubo, K. Inoue, T. Arai and K. Nishii, "Whole body Teleoperation for Humanoid Robot by Marionette System”, Proc. IEEE/RSJ Intl Conf on Intelligent Robotics and Systems (IROS), (2006), pp. 4459-4465,.

[10] N. Miller, O. C. Jenkins, M. Kallman and M. J. Mataric, "Motion capture from inertial sensing for untethered humanoid teleoperation", Proc. of the 4th IEEE-RAS Intl Conf on Humanoid Robotics, (2004), pp. 547 - 565.

[11] H. Song, D. Kim, M. Park and J. Park, "Teleoperation between Human and Robot Arm using Warable Electronic Device", Proceedings of the 17th IFAC World Congress, (2008); Seoul, Korea.

[12] J. Aleotti, A. Skoglund and T. Duckett, "Position Teaching of a Robot Arm by Demonstration with a Wearable Input Device”, Proc Int. Conf on Intelligent Manipulation and Grasping, (IMG04), (2004), pp. 459 - 464.

[13] J. Baillient, "Kinematic Programming Alternatives for Redundant Manipulators", In IEEE Intl. Conf. on Robotics and Automation, (1985), pp. 722 - 728 .

[14] http://www.inct-sec.org/actrep/sites/default/files/facilities/LRM.pdf

[15] R. O'Flaherty, P. Vieira and M. X. Gret, "Humanoid Robot Teleoperation for Tasks with Power Tools", IEEE Int. Conf. on Tech. for practical Robot Application, TEPRA'13, (2013), pp. 1-6.

[16] Information on www.world.honda.com/asimo

[17] T. Liu, H. Utsuomiya, Y. Inoue and K. Shibata, "Synchronous Imitation Control for Biped Robot Based on Wearable Human Motion Analysis System", IEEE/RSJ International Conference on Intelligent Robots and Systems, (2008); France.

[18] T. Liu, Y. Inoue, K. Shibata and H. Morioka, "Development of Wearable Sensor Combinations for Human Lower Extremity Motion Analysis", IEEE International Conference on Robots and Automation, (2006); Florida.

[19] A. Ude, M. Curtis, M. Riley and C. G. Atkeson, "Automatic generation of kinematic models for the conversion of human motion capture data into humanoid robot motion", In Proc. First IEEE-RAS Int. Conf. Humanoid Robots, (2000).

[20] T. Liu, Y. Inoue and K. Shibata, "Imitation Control for Biped Robot Using Wearable Motion Sensor”, Journal of Mechanisms and Robotics, vol. 2, (2010).

[21] R. Chalodhorn, D. B. Grimes, K. Grochow and R. P. N. Rao, "Learning to Walk through Imitation", Twentieth International Joint Conference on Artificial Intelligence, India, (2007).

[22] S. Ha, Y. Bai and C. K. Liu, "Human Motion Reconstruction from Force Sensors", ACM SIGGRAPH Symposium on Computer Animation, (2011).

[23] H. Y. Liu, W. J. Wang, R. J. Wang, C. W. Tung, P. J. Wang and I. P. Chang, " Image Recognition and Force Measurements Application in the Humanoid Robot Imitation”, IEEE Trans. On Instrumentation and Measurement, vol. 61, no. 1, (2012).

[24] Keio University, Walky project, Graduate School of Media design, (2009).

[25] Andre Senior and Sabri Tosunoglu, "Design of a Biped Robot", Florida Conference on Recent Advances in Robotics, FCRAR, (2006).

[26] S. Sternberg, "Pro seminar in Psychological Methods- Reaction - Time Experimentation", Psychology 600-301, Spring Semester, (2004).

[27] J. Peternelj and M. Batista, “The Falling Time of an Inverted Plane Pendulum”, http://arxiv.org/abs/physics/ 0607080.

[28] Duy Le Nguyen and Myung-Eui Lee, "OSEK/VDX Porting to the Teo-Wheel Mobile Robot Based on the Differential Drive Method", Journal of Information and Communication Convergence Engineering, vol. 10, no. 4, pp. 372- 377, (2012).

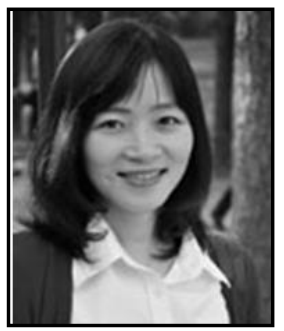

Heejung Byun received the B.S degree from Soongsil University, Korea, in 1999, the M.S. degree from Korea Advanced Institute of Science and Technology (KAIST), Korea, in 2001, and the Ph.D. degree from KAIST in 2005. She was a senior researcher in Samsung Electronics, Ltd. from 2007 to 2010. She is currently a professor with the Department of Information and Telecommunications Engineering, Suwon University, Korea. Her research interests include network protocol, network modeling, controller design, and performance analysis. 


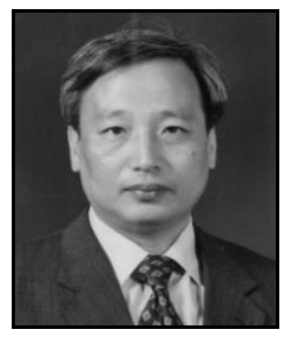

Sugoog Shon received his B.S. (1982) degree in Electrical Engineering from Seoul National University, his M.S. (1984) degree in Electrical Engineering from Seoul National University, and his Ph.D. (1996) degree in Electrical and Computer Engineering from the University of Texas at Austin. $\mathrm{He}$ is a professor in the Department of Information and Telecommunication at the University of Suwon in Korea. His research interests include computer and embedded system, robot, network protocol, network simulation, and network programming. 